\title{
HARMONICITY MODULUS AND APPLICATIONS TO THE APPROXIMATION BY POLYHARMONIC FUNCTIONS
}

\author{
O. I. KOUNCHEV \\ Institute of Mathematics, Bulgarian Academy of Sciences, \\ Acad. G. Bonchev St. 8, 1113 Sofia, Bulgaria
}

\begin{abstract}
In the present paper we introduce the notion of harmonicity modulus and harmonicity $K$-functional and apply these notions to prove a Jackson type theorem for approximation of continuous functions by polyharmonic functions. For corresponding results on approximation by polynomials see [3, 7].

Key words: Harmonicity modulus, $K$-functional, polyharmonic functions, Jackson type theorem.
\end{abstract}

\section{Notions and Notations}

Suppose that $D \subset \mathbb{R}^{n}$ is an open, connected and bounded set $(n \geq 2)$. We shall work with functions $f$ in the space $H C^{r}(D), r \geq 0$, consisting of all functions $f$ such that $\Delta^{r} f$, the $r^{\text {th }}$ power of the Laplacian, exists and is continuous in $\bar{D}$. In the space $H C^{0}(\bar{D})=C(\bar{D})$ of functions which are continuous in $\bar{D}$ the usual norm is

$$
\|f\|:=\max _{x \in \bar{D}}|f(x)| .
$$

By $B(x ; t)$ we will denote an open ball in $\mathbb{R}^{n}$ :

$$
B(x ; t):=\left\{y \in \mathbb{R}^{n}:|x-y|<t\right\} .
$$

For the function $f$, any point $x \in D$, and a sufficiently small positive number $h$ we will consider the spherical mean

$$
\mu_{0}(x, h):=\mu_{0}(f ; x, h):=\frac{1}{\omega_{n}} \int_{\Omega_{\xi}} f(x+h \xi) d \omega_{\xi} ;
$$

here $\Omega_{\xi}$ denotes the unit sphere in $\mathbb{R}^{n}, \omega_{n}$ denotes its area and $d \omega_{\xi}$ is the area element on $\Omega_{\xi}$. 
Further we define the quantity

$$
\Delta_{h}(f ; x):=\mu_{0}(f ; x, h)-f(x) .
$$

Throughout the paper we shall use the symbol $C$ as an universal constant.

\section{Harmonicity Modulus}

DEFINTION 1. The harmonicity modulus of the function $f$ in the domain $D$ is defined by

$$
\omega^{h}(u):=\omega^{h}(f ; u):=\sup \left|\Delta_{t}(f ; x)\right|,
$$

where the sup is taken over $0<t \leq u$, and $B(x ; t) \subset D$.

REMARK. It is clear that

$$
\omega^{h}(f ; u) \leq \omega_{1}(f ; u)
$$

where $\omega_{1}$ is the usual first modulus of continuity (see [5, 9]).

It is easy to see that we have the representation

$$
\Delta_{h}(f ; x)=\frac{1}{2 \omega_{n}} \int_{\Omega}(f(x+h \xi)-2 f(x)+f(x-h \xi)) d \omega_{\xi} .
$$

This implies

$$
\omega^{h}(f ; u) \leq \omega_{2}(f ; u)
$$

for the usual second modulus of continuity (cf. $[5,9])$.

PROPOSITION 1. For every function $f$, continuous in $\bar{D}$, the harmonicity modulus has the following properties:

1. $\lim _{t \longrightarrow 0} \omega^{h}(f ; t)=0$;

2. $\omega^{h}(f ; u)$ is a monotone increasing funciton;

3. for every positive $u$ the inequality

$$
\omega^{h}(f+g ; u) \leq \omega^{h}(f ; u)+\omega^{h}(g ; u)
$$

holds;

4. for every positive number $u$ the inequality

$$
\omega^{h}(f ; u) \leq 2\|f\|
$$

holds.

Proof. Property 1) follows from the definition of $\mu_{0}$ and the continuity of the function $f$. Properties 2) and 3) are evident. Property 4) follows from the easy-to-check representation

$$
\Delta_{h}(f ; x)=\frac{1}{\omega_{n}} \int_{\Omega}(f(x+h \xi)-f(x)) d \omega_{\xi} .
$$


Let us introduce the integral operator $J_{0}$ by

$$
J_{0}[\phi ; R]:=\int_{0}^{R}\left(r-r^{n-1} R^{-n+2}\right) \phi(r) d r
$$

for $n \geq 3$, and by

$$
J_{0}[\phi ; R]:=\int_{0}^{R} r \log \left(\frac{R}{r}\right) \phi(r) d r
$$

for $n=2$.

Further we will need different forms of the classical Pizzetti formula for the representation of the spherical means (see $[2,8]$ ).

THEOREM 1. Let the function $f$ have a continuous Laplacian $\Delta f$ in the domain D. Then the following representation holds:

$$
\mu_{0}(f ; x, R)=f(x)+l_{n} J_{0}\left[\mu_{0}(\Delta f ; x, \cdot) ; R\right],
$$

where $l_{n}=\frac{1}{n-2}$ for $n \geq 3$ and $l_{2}=1$.

The remainder can also be written as

$$
J_{0}\left[\mu_{0}(\Delta f ; x, \cdot) ; R\right]=\mu_{0}(\Delta f ; x, \vartheta R) J_{0}[1, R]
$$

with some number $\vartheta=\vartheta(x ; R)$ such that $0<\vartheta<1$. Since $J_{0}[1 ; R]=c_{n} R^{2}$, where $c_{2}=1 / 4, c_{n}=\frac{n-2}{2 n}$ for $n \geq 3$, we have the representation

$$
\mu_{0}(f ; x, R)=f(x)+d_{n} R^{2} \Delta f(\xi),
$$

where the opint $\xi=\xi(x, R) \in B(x ; R)$ and $d_{n}=c_{n} l_{n}=\frac{1}{2 n}$.

PROPOSITION 2. Suppose that the function $f$, defined and continuous in $\bar{D}$, has a continuous Laplacian $\Delta f$ in $\bar{D}$ satisfying the inequality

$$
|\Delta f(x)| \leq M, \quad x \in \bar{D} .
$$

Then the following inequality holds for every positive number u:

$$
\omega^{h}(f ; u) \leq M d_{n} u^{2} .
$$

The proof follows immediately from Pizzetti's formula (8).

The harmonicity modulus plays a role similar to that of the second modulus of continuity in the one-dimensional case (see [3]). This is well seen from the following classical result (cf. [8]).

THEOREM 2. Let $u$ be a function defined and integrable in the domain $D$ in $R^{n}$. Then, if $t>0$, we have

$$
\omega^{h}(f ; t)=0
$$


if and only if $f$ is harmonic in D, i.e.

$$
\Delta f(x)=0, \quad x \in D .
$$

Theorem 2 is the motivation for calling $\omega^{h}$ the harmonicity modulus. We also recall that harmonic functions are considered to be a multivariate analogue to the linear functions in one dimension.

\section{Harmonicity $K$-Functional}

Here we introduce the notion of harmonicity $K$-functional which provides a basic tool for studying the important properties of the harmonicity modulus.

DEFINITION 2. For every function $f \in C(\bar{D})$ and every number $t>0$ we define the harmonicity $K$-functional by

$$
K^{h}(f ; t):=\inf \left\{\|f-g\|+t^{2}\|\Delta g\|\right\},
$$

where the infimum is taken over all functions $g \in H C^{1}(\bar{D})$.

\section{Harmonicity Modulus and Harmonicity $\mathrm{K}$-Functional}

The main technical result of the paper is proved in the present and the next sections. Roughly speaking, it states that the harmonicity modulus and the harmonicity $K$-functional are equivalent on compact subdomains of D.

The simple part of the equivalence is the following

LEMMA 1. Let $D$ be an open set in $\mathbb{R}^{n}$. For all $t$ with $0<t<\infty$ and $f \in C(\bar{D})$, the inequality

$$
\omega^{h}(f ; t) \leq C K^{h}(f ; t)
$$

holds with some constant $C>0$.

Proof. The proof is based on a standard argument. We split $f=f-g+g$, and apply Propositions 1 and 2 to obtain the inequality

$$
\begin{aligned}
\omega^{h}(f ; t) & \leq \omega^{h}(f-g ; t)+\omega^{h}(g ; t) \leq 2\|f-g\|+d_{n} t^{2}\|\Delta g\| \\
& \leq \max \left\{2, d_{n}\right\}\left[\|f-g\|+t^{2}\|\Delta g\|\right] .
\end{aligned}
$$

Since $g \in H C^{1}(\bar{D})$ is arbitrary, the statement (10) is proved.

The domination of $K^{h}$ by $\omega^{h}$ will be established only on compact subdomains of $D$ in the sense that the $K$-functional of the subdomain $D_{1}, K_{d_{1}}^{h}$ will be proved 
to be dominated by the harmonicity modulus $\omega_{D}^{h}$ with respect to the domain $D$.

The problem is that for every $R$ (possibly such that $R<R_{1}$ for some sufficiently small positive number $R_{1}$ ) we have to find a function $g_{R} \in H C^{1}(\bar{D})$ such that

$$
\left\|f-g_{R}\right\|+R^{2}\left\|\Delta g_{R}\right\| \leq C \omega^{h}(f ; R),
$$

where the constant $C$ does not depend on $f$ and $R$.

Following the scheme given in [5], taking some spherical means of the function $f$, we succeed in constructing the function $g_{R}$ not on the whole of $D$ but on every subdomain $D_{1}$, such that $\overline{D_{1}} \subset D$ and $R_{1} \leq \operatorname{dist}\left(D_{1}, \partial D\right)$. In such a way we can prove the inequality (11) over subdomains where the norm $\|\cdot\|$ is in fact $\|\cdot\|_{D_{1}}$.

\section{Domination of $K^{h}$ by $\omega^{h}$ on Compact Subdomains}

Having in mind Pizzetti's formula (7) in Theorem 1, we consider the function

$$
g_{R, t}(x)=v(t) J_{0, s}\left[\mu_{0}(f ; x, R s) ; t\right] .
$$

Here $J_{0}$ is the operator given by (5) and (6) and $J_{0, s}$ means that $s$ is the input variable for $J_{0}$; the output variable is $t ; v(t)$ is equal to $\left(J_{0}[1 ; t]\right)^{-1}$, where $J_{0}[1 ; t]$ is the value of the functional for $\phi(t)=1$, so in fact $\frac{1}{v(t)}=t^{2}\left(\frac{1}{2}-\frac{1}{n}\right)$ for $n \geq 3, \frac{1}{v(t)}=\frac{t^{2}}{4}$ for $n=2$.

The operator $J_{0}$ changes the output in a specific way described by

PROPOSITION 3. For every integrable function $\phi$ and positive numbers $s$ and $R$ we have

$$
J_{0, s}[\phi(s t) ; R]=\frac{1}{s^{2}} J_{0, t}[\phi(t) ; s R] .
$$

Proof. We give the proof for $n \geq 3$. Then $J_{0}$ is given by formula (5). The case $n=2$ is similar.

By changing the variables we obtain

$$
\begin{aligned}
J_{0, s}[\phi(s t) ; R] & =\int_{0}^{R}\left(t-t^{n-1} R^{-n+2}\right) \phi(s t) d t \\
& =\frac{1}{s^{2}} \int_{0}^{s R}\left(t-t^{n-1}(s R)^{-n+2}\right) \phi(t) d t \\
& =\frac{1}{s^{2}} J_{0, t}[\phi(t) ; s R] .
\end{aligned}
$$

Proposition 3 shows that (12) becomes

$$
g_{R, t}(x)=v(t) \frac{1}{R^{2}} J_{0}\left[\mu_{0}(f ; x, \cdot) ; t R\right] .
$$


The following is the main technical result of the paper.

THEOREM 3. For every subdomain $D_{1}$ such that $\overline{D_{1}} \subset D$, the inequality

$$
K^{h}(f ; R)_{D_{1}} \leq C \omega^{h}(f ; R)_{D}
$$

holds for every number $R$ with

$$
0<R<d=\operatorname{dist}\left(D_{1}, \partial D\right)
$$

here the constant $C$ does not depend on $f$ and $R$, and $K^{h}(f ; R)_{D_{1}}$ denotes the harmonicity $K$-functional for the domain $D_{1}$, while $\omega^{h}(f ; R)_{D}$ denotes the harmonicity modulus on $D$.

Proof. Let us notice first that the function $g_{R, t}$ given by (12), is well defined in $D_{1}$ for $R$ satisfying (16) and every number $t$ with $0<t<1$.

Since $v(t) J_{0}[1 ; t]=1$, we obtain

$$
g_{R, t}(x)-f(x)=v(t) J_{0, s}\left[\mu_{0}(f ; x, R s)-f(x) ; t\right]
$$

for every number $R$ satisfying (16) and every number $t$ with $0 \leq t \leq 1$. Hence, for every $x \in D_{1}$ we obtain the inequalities

$$
\left|g_{R, t}(x)-f(x)\right| \leq v(t) J_{0}[1 ; t] \omega^{h}(f ; R t) \leq \omega^{h}(f ; R) .
$$

Consequently, we have proved the domination of the first term of $K^{h}$ by $\omega^{h}$ :

$$
\left\|g_{R, t}-f\right\| \leq \omega^{h}(f ; R) .
$$

For proving the domination of the second term, we will check the value of $\Delta g_{R, t}(x)$ for $x \in D_{1}$.

First let us suppose that $f$ is twice differentiable in $D$, i.e. $f \in C^{2}(D)$. By formula (14) the Laplacian of $g_{R, t}(x)$ is then equal to

$$
\Delta g_{R, t}(x)=v(t) \frac{1}{R^{2}} J_{0, s}\left[\mu_{0}(\Delta f ; x, s) ; t R\right] .
$$

Hence, combining with formula (7), we obtain

$$
\Delta g_{R, t}(x)=v(t) \frac{1}{l_{n} R^{2}}\left[\mu_{0}(f ; x, R t)-f(x)\right] .
$$

This implies the inequalities

$$
R^{2}\left|\Delta g_{R, t}(x)\right| \leq v(t) \frac{1}{l_{n}} \omega^{h}(f ; t R) \leq C \omega^{h}(f ; R)
$$

for every $x \in D_{1}$ and every $R<\operatorname{dist}\left(D_{1}, \partial D\right)$, where $C$ is a constant given by

$$
C:=\left|v\left(t_{1}\right)\right| / \not_{n}
$$

and $t_{1}$ is an arbitrary number with $0<t_{1}<1$ satisfying $v\left(t_{1}\right) \neq 0$. 
Inequality (20) implies that the second term of $K^{h}$ is dominated by $\omega^{h}$. This ends the proof for $f \in C^{2}(D)$.

In the case of an arbitrary continuous function $f$, let us take an approximation to $f$, say $f_{\delta} \in C^{\infty}, \delta>0$, such that $f_{\delta}$ converges to $f$ uniformly on $D_{1}$ $\delta \longrightarrow 0$ (see this construction in [1, paragraph 5$]$ ).

Since $\overline{D_{1}}$ is a compact set and $f$ is continuous in $D$, we obtain by a standard limiting argument that formula (7) takes for an arbitrary integrable function $f$ the form

$$
\mu_{0}(f ; x, R)=f(x)+l_{n} \Delta J_{0}\left[\mu_{0}(f ; x, \cdot) ; R\right] .
$$

This implies that the relations (19) and (20) hold as well.

Now we are ready to prove an important property of the harmonicity modulus.

For an arbitrary subdomain $D_{1}$ of $D$ let us denote by $\omega^{h}(\cdot ; \cdot)_{D_{1}}$ the harmonicity modulus for the set $D_{1}$.

THEOREM 4. For every subdomain $D_{1}$ of $D$ such that $\overline{D_{1}} \subset D$, the following inequalities hold:

$$
\omega^{h}(f ; \lambda R)_{D_{1}} \leq C(\lambda+1)^{2} \omega^{h}(f ; r)_{D}
$$

for every number $R \leq d=\operatorname{dist}\left(D_{1}, \partial D\right)$ and every number $\lambda>0$ such that $\lambda R \leq d ;$

(ii)

for all positive real numbers $a$ and $b$.

$$
\omega^{h}(f ; a+b)_{D_{1}} \leq C\left[\omega^{h}(f ; a)_{D}+\omega^{h}(f ; b)_{D}\right]
$$

Proof. Inequality (i) follows from a similar inequlity for the harmonicity $K$ functional. Indeed, since

$$
\|f-g\|_{D_{1}}+(\lambda u)^{2}\|\Delta g\|_{D_{1}} \leq(\lambda+1)^{2}\left(\|f-g\|_{D_{1}}+u^{2}\|\Delta g\|_{D_{1}}\right)
$$

for an arbitrary function $g \in H C^{1}\left(\overline{D_{1}}\right)$, by the definition of the harmonicity $K$-functional we obtain the inequality

$$
K^{h}(f ; \lambda u)_{D_{1}} \leq(\lambda+1)^{2} K^{h}(f ; u)_{D_{1}},
$$

for every number $\lambda \geq 0$.

Lemma 1 gives

$$
\omega^{h}(f ; \lambda R)_{D_{1}} \leq C K^{h}(f ; \lambda R)_{D_{1}},
$$

and Theorem 3 implies

$$
K^{h}(f ; \lambda u)_{D_{1}} \leq C \omega^{h}(f ; R)_{D}
$$

for every number $R<d$. These inequalities imply the inequality (i).

In order to prove inequality (ii) let us note that

$$
(a+b)^{2} \leq 2\left(a^{2}+b^{2}\right)
$$


for all real numbers $a$ and $b$. This implies

$$
\begin{aligned}
& \|f-g\|_{D_{1}}+(a+b)^{2}\|\Delta g\|_{D_{1}} \\
& \leq\|f-g\|_{D_{1}}+2\left(a^{2}+b^{2}\right)\|\Delta g\|_{D_{1}} \\
& \leq 2\|f-g\|_{D_{1}}+2 a^{2}\|\Delta g\|_{D_{1}}+2\|f-g\|_{D_{1}}+2 b^{2}\|\Delta g\|_{D_{1}} .
\end{aligned}
$$

The definition of $K^{h}$ implies the inequality

$$
K^{h}(f ; a+b)_{D_{1}} \leq 2\left(K^{h}(f ; a)_{D_{1}}+K^{h}(f ; b)_{D_{1}}\right) .
$$

Now inequality (ii) follows by arguments similar to those used for (i).

\section{Polyharmonic Kernels}

Here we introduce kernels which are polyharmonic functions and arise naturally from the Jackson type kernels used in approximation theory [3].

Let us recall that the function $f$ is called polyharmonic of order $p$ in an open set $D$, where $p$ is a nonnegative integer, if it satisfies the equation

$$
\Delta^{p} f(x)=0 \quad \text { for } x \in D ;
$$

here the iterated Laplacian of order $p$ is defined inductively by the equations $\Delta^{k+1}:=\Delta \Delta^{k}$ for $k \geq 0$ and $\Delta^{0}:=i d$ (see [8]).

Let us remind the notion of Jackson type kernel (cf. [3]).

DEFINITION 3. A kernel of Jackson type of order $\nu$, where $\nu=1,2, \ldots$, is defined to be the function given by

$$
J_{k ; \nu}(t):=\left(\gamma_{k, \nu}\right)^{-1}[\sin (\nu t / 2) / \sin (t / 2)]^{2 k},
$$

where $k$ is a natural number and the constant is

$$
\gamma_{k, \nu}:=\frac{1}{\pi} \int_{-\pi}^{\pi}[\sin (\nu t / 2) / \sin (t / 2)]^{2 k} d t .
$$

For the properties of these kernels we refer to [3]. Through the substitution

$$
x=2 \sin (t / 2), \quad t \in[-\pi, \pi], \quad x \in[-2,2],
$$

we obtain the nonperiodic Jackson type kernels:

$$
\bar{J}_{k, \nu}(x)=\gamma_{k, \nu}\left(\bar{\gamma}_{k, \nu}\right)^{-1} J_{k, \nu}\left[\arccos \left(1-x^{2} / 2\right)\right] ;
$$

here the constant is

$$
\bar{\gamma}_{k, \nu}:=\int_{-1}^{1} \gamma_{k, \nu} J_{k, \nu}\left[\arccos \left(1-x^{2} / 2\right)\right] d x,
$$


for $\nu \in \mathbb{N}$.

Finally, we define the polyharmonic Jackson type kernels of order $p$ by the equation

$$
\widetilde{J}_{k, p}(x):=\widetilde{J}_{k, p}(|x|)=\bar{\gamma}_{k, p}\left(\widetilde{\gamma}_{k, p}\right)^{-1} \bar{J}_{k, p}(|x|),
$$

for $p \in \mathbb{N}$, and for every $x \in \mathbb{R}^{n}$ such that $|x| \leq 2$; here the constant is given by

$$
\widetilde{\gamma}_{k, p}=\int_{0}^{1} r^{n-1} \bar{\gamma}_{k, p} \bar{J}_{k, p}(r) d r=\int_{0}^{1} r^{n-1}[\sin (\nu t / 2) / \sin (t / 2)]^{2 k} d r,
$$

where $t=\arccos \left(1-r^{2} / 2\right)$.

THEOREM 5. The polyharmonic Jackson type kernels have the following properties:

(i) For all natural numbers $p$ and $k$, the kernel $\widetilde{J}_{k, p}$ is a nonnegative polyharmonic function of order $k(p-1)+1$ and $\widetilde{J}_{k, p}(x)$ is defined for every $x \in \mathbb{R}^{n}$ satisfying $|x| \leq 2$;

(ii) $\int_{B(0 ; 1)} \widetilde{J}_{k, p}(x)=1$;

(iii) If $I_{i}$ is defined by

$$
I_{i}:=\int_{0}^{1} t^{i+n-1} \widetilde{J}_{k, p}(t) d t
$$

for nonnegative integers $i$, then for $i<2 k-n$ we have the inequality

$$
I_{i} \leq C p^{-i},
$$

and for $i=2 k-n$ we have the inequality

$$
I_{i} \leq C(\ln p) p^{-i} .
$$

The proof of Theorem 5 is based on standard arguments [3] and is given in detail in a forthcoming paper [6].

\section{A Direct Theorem of Jackson Type}

Here we prove an approximation theorem which is analogous to the direct theorem of Jackson for the approximation by polynomials in the one-dimensional case, where the rate of approximation is estimated by the first and the second modulus of continuity (see $[3,7]$ ).

In the multivariate case we approximate by polyharmonic functions and the rate of approximation is estimated by the harmonicity modulus.

Let us first give some necessary notations. Let $K$ be a polyharmonic function on $\left\{x \in \mathbb{R}^{n}:|x| \leq 1\right\}$. Then for every function $f$ defined and continuous in the domain $D$, we can define the operator

$$
T_{K}[f](x):=\int_{B(x ; 1)} K(x-u) f(u) d u
$$


for every $x \in D$ such that $\operatorname{dist}(x, \partial D)<1$.

Let the domain $D$ be regular in the sense of solvability of the Dirichlet problem (see [4]), and let the function $f$ be continuous in $\bar{D}$. Then there exists a harmonic function $h_{f}$ solving the Dirichlet problem in $D$, i.e.

$$
\begin{aligned}
\Delta h_{f}(x) & =0 \quad \text { for } x \in D, \\
h_{f}(x) & =f(x) \quad \text { for } x \in \partial D .
\end{aligned}
$$

We shall consider the function $F_{0}$ given by the following conditions:

$$
F_{0}(x):=f(x)-h_{f}(x) \quad \text { for } x \in \bar{D},
$$

and

$$
F_{0}(x):=0 \quad \text { for } x \notin \bar{D} .
$$

The function $F_{0}$ is evidently continuous on the whole space and it makes sense to consider its harmonicity modulus there or in domains containing $D$.

Another interesting feature of the function $F_{0}$ is that its harmonicity modulus in $D$ satisfies

$$
\omega^{h}\left(F_{0} ; t\right)_{D}=\omega^{h}(f ; t)_{D} .
$$

This follows immediately from the Gauss mean value theorem, which states that

$$
\mu_{0}\left(h_{f} ; x, t\right)=h_{f}(x)
$$

for every $x \in D$ and $t>0$ such that $B(x ; t) \subset D$.

Notice that for every domain $D_{1}$ such that $\bar{D} \subset D_{1}$ we have

$$
\omega^{h}\left(F_{0} ; t\right)_{D_{1}}=\omega^{h}\left(F_{0} ; t\right)_{\mathbb{R}^{n}}
$$

for every positive number $t \leq \operatorname{dist}\left(D, \partial D_{1}\right)$. Here $\omega^{h}\left(F_{0} ; t\right)_{\mathbb{R}^{n}}$ denotes the harmonicity modulus of the function $F_{0}$ in the whole space.

Let $D_{2}$ be a domain such that $\overline{D_{1}} \subset D_{2}$. Then we can apply Theorem 3 to obtain the following inequalities

$$
C_{1} \omega^{h}\left(F_{0} ; t\right)_{D} \leq K^{h}\left(F_{0} ; t\right)_{D} \leq C_{2} \omega^{h}\left(F_{0} ; t\right)_{D_{2}}
$$

for sufficiently small numbers $t>0$ and appropriate constants $C_{1}, C_{2}$ which do not depend on $f$ and $t$.

Next suppose that, for some nonnegative $r$, the function $f$ is in $H C^{r}(\bar{D})$. Then, inductively in $r$, we obtain a solution $h_{f}$ to the following boundary value problem:

$$
\begin{aligned}
\Delta^{r+1} h_{f}(x) & =0, & x \in D ; \\
\Delta^{j} h_{f}(x) & =\Delta^{j} f(x), & x \in \partial D,
\end{aligned}
$$

for $j=0,1, \ldots, r$. 
We shall consider the function $F_{r}$ given by

$$
\begin{array}{lr}
F_{r}(x):=f(x)-h_{f}(x) & \text { for } x \in \bar{D} ; \\
F_{r}(x):=0 & \text { for } x \notin \bar{D} .
\end{array}
$$

Note that the function $F_{r}$ is continuous on the whole space together with $\Delta^{r} F_{r}$ and we can apply to it all properties of the harmonicity modulus, a fact which will be used below.

Now we are ready to state the following result which is the main application of the harmonicity modulus in the present paper.

THEOREM 6. Let the domain $D$ be regular in the sense of solvability of the Dirichlet problem. Let for some integer $r \geq 0$, the function $f \in H C^{r}(\bar{D})$. Let us denote by $F_{r}$ the function given by (23). Then, for every natural number $p$ satisfying $p \geq r+1$, there exists a polyharmonic function $T_{p}$ of order $p$ in $D$ satisfying the inequality

$$
\left|f(x)-T_{p}(x)\right| \leq C \omega^{h}\left(\Delta^{r} F_{r} ; \frac{1}{p}\right) \frac{1}{p^{2 r}}
$$

for every $x \in \bar{D}$, where the constant $C>0$ depends on the domain $D$ and on $r$. Proof. (1) By a similarity transform we can suppose that the domain $D$ is contained in the ball $B(0 ; 1 / 2)$. Obviously, this transform preserves the polyharmonic functions. To find the harmonicity modulus for the function $G(x)=$ $f(\lambda x)$, where $\lambda$ is a positive real number, let us compute the harmonicity difference given by (2):

$$
\Delta_{t}(G ; x)=\mu_{0}(G ; x, t)-G(x)=\mu_{0}(f ; \lambda x ; \lambda t)-f(\lambda x)=\Delta_{\lambda t}(f ; \lambda x) .
$$

Hence, we obtain

$$
\omega^{h}(G ; t)_{D}=\omega^{h}(f ; \lambda t)_{\lambda D},
$$

where $\lambda D$ is the domain given by

$$
\lambda D=\left\{y \in \mathbb{R}^{n}: y=\lambda x, \quad x \in D\right\} .
$$

So for a domain $D_{1}$ such that $\overline{D_{1}} \subset D$ we have

$$
\omega^{h}(G ; t)_{D_{1}}=\omega^{h}(f ; \lambda t)_{\lambda D_{1}},
$$

which proves the inequality

$$
\omega^{h}(G ; t)_{D_{1}} \leq(\lambda+1)^{2} \omega^{h}(f ; t)_{\lambda D}
$$

for every number $t \leq \operatorname{dist}\left(\lambda D_{1}, \partial \lambda D\right)$.

This shows that the harmonicity modulus is at most multiplied by a constant as a result of a similarity transform. Applied to the modulus $\omega^{h}\left(\Delta^{r} F_{0} ; p^{-1}\right)_{\mathbb{R}^{n}}$ we see that by (22) it only changes up to a constant multiple. 
(2) We will define the polyharmonic function $T_{p}(x)=T_{p}(f ; r, x)$ of order $p$ inductively by the following recurrency relation:

$$
\begin{aligned}
T_{p}(x) & :=T_{p}\left(F_{r} ; m, x\right) \\
& :=T_{p}\left(F_{r} ; m-1, x\right)+T_{k, \nu}\left[F_{r}(\cdot)-T_{p}\left(F_{r} ; m-1, \cdot\right)\right](x),
\end{aligned}
$$

for every $x \in \bar{D}$ and every $m$ with $1 \leq m \leq r$.

Here $T_{k, \nu}$ is a short notation for the operator given by formula (21) for the Jackson Type kernel $\widetilde{J}_{k, \nu}$, where we take $k$ big enough to satisfy $2 k-n \geq 3$, and put $\nu:=[(p-1) / k]+1$ (here $[y]$ denotes, as usually, the greatest integer which does not exceed $y$ ). The choice of such $\nu$ provides that the order of the polyharmonic function $\widetilde{J}_{k, p}$ be equal to $k(\nu-1)+1<p$.

Note that the operator $T_{k, \nu}$ is well defined and produces a polyharmonic function since $F_{r}$ is a finite function, the kernels are defined in $B(0 ; 1)$ and we have the inclusion $D \subset B(0 ; 1 / 2)$.

(3) Let us check the Theorem for $r=0$. In this case we have $f \in C(\bar{D})$. Due to Theorem 5 the following holds:

$$
\begin{aligned}
D(x) & :=R_{0}(x)-T_{k, \nu}\left[F_{0}\right](x) \\
& =\int_{B(x ; 1)}\left[F_{0}(x)-F_{0}(u)\right] \widetilde{J}_{k, \nu}(x-u) d u \\
& =\int_{0}^{1}\left\{\int_{\Omega_{\xi}}\left[F_{0}(x)-F_{0}(x-r \xi)\right] d \omega_{\xi}\right\} r^{n-1} \widetilde{J}_{k, \nu}(r) d r .
\end{aligned}
$$

By the properties of the harmonicity modulus (see Theorem 4) this gives the following estimate

$$
\begin{aligned}
|D(x)| & \leq \omega_{n} \int_{0}^{1} r^{n-1} \widetilde{J}_{k, \nu}(r) \omega^{h}\left(F_{0} ; r\right)_{\mathbb{R}^{n}} d r \\
& \leq C \omega^{h}\left(F_{0} ; p^{-1}\right)_{\mathbb{R}^{n}} \omega_{n} \int_{0}^{1} r^{n-1} \widetilde{J}_{k, \nu}(r)(p r+1)^{2} d r
\end{aligned}
$$

for every $p \geq 1$ and some constant $C>0$.

Again, applying Theorem 5, (iii), since $2 k-n \geq 3$, we have the inequality

$$
\int_{0}^{1} r^{n-1} \widetilde{J}_{k, \nu}(r)(p r+1)^{2} d r \leq C \int_{0}^{1} r^{n-1} \widetilde{J}_{k, \nu}(r)(\nu r+b r+1)^{2} d r \leq C_{1}
$$

for appropriate constants $C, C_{1}$ and $b$.The last gives, finally, that

$$
|D(x)| \leq C \omega^{h}\left(F_{0} ; p^{-1}\right), \quad x \in \bar{D},
$$

for some constant $C>0$. From this estimate we get the statement for $r=0$.

(4) Before proceeding by induction on $r$, let us note the following. If for some function $\phi$ on some domain $D$, such that $\Delta \phi$ is continuous on $D$, the inequality

$$
|\Delta \phi(x)| \leq M, \quad x \in D
$$


holds, then by Proposition 2 we obtain the inequality

$$
\omega^{h}(\phi ; t) \leq M d_{n} t^{2}
$$

for every number $t>0$. Hence, by (26), we obtain the inequality

$$
\left|\phi(x)-T_{k, \nu}[\phi](x)\right| \leq C M \frac{1}{p^{2}}
$$

for an appropriate constant $C$.

(5) Let us suppose that the Theorem is true for the classes of functions $H C^{0}, H C^{1}, \ldots, H C^{r}, r \geq 0$. Then, if $f \in H C^{r+1}$, it follows that $\Delta f \in H C^{r}$, and equality (25) implies that

$$
\Delta T_{p}\left(F_{r+1} ; r, x\right)=T_{p}\left(\Delta F_{r+1} ; r, x\right) .
$$

Applied to the function $\Delta F_{r+1}$, the induction hypothesis (24) gives

$$
\begin{aligned}
\left|\Delta\left[F_{r+1}(x)-T_{p}\left(F_{r+1} ; r, x\right)\right]\right| & =\left|\Delta F_{r+1}(x)-T_{p}\left(\Delta F_{r+1} ; r, x\right)\right| \\
& \leq C \omega^{h}\left(\Delta^{r+1} F_{r+1} ; p^{-1}\right)_{D} \times p^{-2 r} .
\end{aligned}
$$

Let us put

$$
\phi(x):=F_{r+1}(x)-T_{p}\left(F_{r+1} ; r, x\right)
$$

and apply inequality (27) to this function $\phi$. We obtain the following inequalities:

$$
\begin{aligned}
& \left|\phi(x)-T_{k, \nu}[\phi](x)\right| \\
& =\left|F_{r+1}(x)-T_{p}\left(F_{r+1} ; r, x\right)-T_{k, \nu}\left[F_{r+1}(\xi)-T_{p}\left(F_{r+1} ; r ; \xi\right)\right](x)\right| \\
& \leq C C_{1} p^{-2} \omega^{h}\left(\Delta^{r+1} F_{r+1} ; p^{-1}\right)_{D} \frac{1}{p^{2 r}} \\
& =C C_{1} p^{-2} \omega^{h}\left(\Delta^{r+1} F_{r+1} ; p^{-1}\right)_{D} \frac{1}{p^{2(r+1)}} .
\end{aligned}
$$

On the other hand, by (25) we have

$$
\phi(x)-T_{k, \nu}(\phi)(x)=F_{r+1}(x)-T_{p}\left(F_{r+1} ; r+1, x\right),
$$

which shows that the inequality in (28) is exactly inequality (24) for $r+1$. This yields the statement of the Theorem for $r+1$.

COROLLARY. In view of the Remark after Definition 1, in Theorem 6 we can replace inequality (24) by the following inequalities

$$
\left|f(x)-T_{p}(x)\right| \leq C \omega_{1}\left(\Delta^{r} F_{r} ; \frac{1}{p}\right) \frac{1}{p^{2 r}}
$$

or

$$
\left|f(x)-T_{p}(x)\right| \leq C \omega_{2}\left(\Delta^{r} F_{r} ; \frac{1}{p}\right) \frac{1}{p^{2 r}}
$$

for $x \in \bar{D}$, where $\omega_{1}$ and $\omega_{2}$ are the usual first and second moduli of continuity (see [5]). 


\section{Acknowledgements}

The author wishes to thank Professor Walter K. Hayman and the reviewer for their invaluable remarks.

\section{References}

1. Besov, O. V., V. P. Hin and S. M. Nikolskii: Integral Representation of Functions and Imbedding Theorems, Nauka, Moskow 1975.

2. Courant, R. and D. Hilbert: Methoden der mathematischen Physik II, Springer, Berlin-Heidelberg-New York 1968.

3. Dzyadyk, V. K.: Introduction to the Theory of Uniform Approximation of Functions by Polynomials, Nauka, Moscow 1977.

4. Helms, L. L.: Einfiihrung in die Potentialtheorie, de Gruyter, BerlinNew York 1973.

5. Johnen, H., and K. Scherer: On the equivalence of the A"-functional and moduli of continuity and some applications, in: Constr. Theory of Funct. of Several Variables, Lecture Notes Math. 571, pp. 119-140, Springer, BerlinHeidelberg-New York (1977).

6. Kounchev, O. I.: Harmonicity moduli and Jackson type theorems for the approximation through polyharmonic functions (preprint).

7. Meinardus, G.: Approximation of Functions: Theory and Numerical Methods, Springer, Berlin-Heidelberg-New York 1967.

8. Nicolescu, M.: Opera Matematica. Functii Poliarmonice, Editura Academiei, Bucuresti 1980.

9. Nikolskii, S. M.: Approximation of Functions of Several Variables and Imbedding Theorems, Springer, Berlin-Heidelberg-New York 1975.

Received September 16, 1991. 